\title{
LOS DERECHOS SEXUALES EN LA ESCUELA: ESCUCHA, DINÁMICA Y ENCUENTRO UNA EXPERIENCIA DE INVESTIGACIÓN ACCIÓN PARIICIPAIIVA'
}

\section{SEXUAL RIGHTS AT SCHOOL: LISTENING, DYNAMICS AND ENCOUNTER A PARTICIPATORY ACTION RESEARCH EXPERIENCE}

\section{Diana Catalina Arango ${ }^{2}$}

RESUMEN Este texto reflexiona sobre una experiencia de Investigación Acción Participativa (IAP) que surge de la Secretaria de Extensión de la Universidad de San Martín, a partir de la relación entre la universidad y su escuela técnica. La creación de un taller de género para trabajar con mujeres adolescentes, con el fin de brindar contenidos sobre derechos sexuales y reproductivos, violencia de género y diversidad sexual, entre otros, guia la propuesta de trabajo, la cual busca que las estudiantes hagan uso de estas herramientas para cuestionar los valores sociales que condicionan a la mujer a un ámbito restringido de la femineidad.

Desde esta relación, lejos de impartir una serie de contenidos, la universidad asume una postura de escucha activa hacia las desigualdades de género y hacia las preguntas que se hacen las adolescentes para comprender su entorno sus necesidades y sus deseos, iniciando un diálogo de saberes en el que la universidad y el territorio no se encuentren escindidos. Palabras claves: Adolescencia, género, derechos sexuales, investigación acción participativa.

1 Artículo recibido el 22 de marzo de 2019. Aceptado el 30 de agosto de 2019.

Esta experiencia se inscribe en el marco del programa Orlando Fals Borda, del Instituto de Altos Estudios Sociales (ldaes) de la Universidad de San Martín (Unsam), en su relación con su Escuela Técnica en 2016 y 2017.

${ }^{2}$ Doctoranda en Sociología, Magister en Sociología económica Idaes-Unsam. Politóloga por la Universidad de Antioquia. Correo electrónico: katia.arango@ gmail.com.

\footnotetext{
${ }^{3}$ Este taller se realizó en compañía de Belén López, estudiante de Antropología del ldaes, compañera del grupo de estudio Géneros, sexualidades y violencias, y del programa contra la violencia de género de la universidad.
}

${ }^{4}$ Ver: Comte, A. (1973). Curso de Filosofía positiva. Buenos Aires: Aguilar.
La adolescencia es una etapa de la vida en la que se concentran ciertas representaciones sociales que oscilan entre la peligrosidad, la depresión, la inestabilidad emocional y el riesgo. Se suele considerar que los y las jóvenes carecen de información y que requieren de la contención y de la seguridad que puedan transferirles las y los adultos. La academia no está exenta de estas representaciones, pero tiene a su favor la misión de hacerse preguntas, de alli que nos propusiéramos conocer cuál era la información que tenían las jóvenes acerca de la violencia de género, la salud sexual y sus dudas. Nos acercamos a la escuela técnica, perteneciente a la universidad, con el fin de investigar y compartir contenidos sobre salud sexual y género con las jóvenes. Nuestra procedencia surgida de un programa de extensión de la Facultad de Ciencias Sociales y del Programa contra la violencia de género habilitó la intersección entre los temas de investigación personal -que en mi caso corresponde al área de la política sexual- y las problemáticas propias del territorio en el que se asienta la universidad ${ }^{3}$. Este enfoque de trabajo permitió un diálogo entre tres lenguajes -el académico, el activista y el de las adolescentes.

La creación del vínculo no es sencilla de construir ya que existe un considerable número de mediaciones necesarias para que se dé, esto es, vínculos barriales con las y los directivos, y con las y los docentes que muchas veces se corresponden con prejuicios, desconfianzas y prevenciones entre la "universidad", "la escuela" y el "barrio". Estos prejuicios provienen de experiencias previas en las que, en primer lugar, no todos los y las investigadores se encuentran interesados en la construcción de dicho vínculo. Algunas tradiciones investigativas consideran que entre investigador e investigado no debe existir una relación estrecha para no pervertir la objetividad de la investigación. Los criterios de avaloratividad investigativa y de la separación entre sujeto investigador y objeto investigado fueron transversales a las Ciencias Sociales del siglo XIX en Europa ${ }^{4}$. Conociendo esta postura, quisimos fijar una diferencia radical como equipo de extensión-investigación, optando por una teoría propia de nuestros contextos, la IAP, metodología de investigación ampliamente trabajada en Colombia por su referente, Orlando Fals Borda, así como los aportes de 
De Prácticas y discursos

Universidad Nacional del Nordeste

Centro de Estudios Sociales

Año 8, Número 12, 2019, Octubre

ISSN 2250-6942

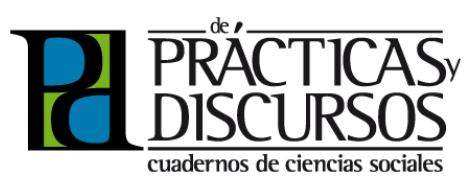

Paulo Freire en materia de educación popular. Esta manera de entender la investigación nos posiciona en un lugar de diálogo de saberes en el que la fuente de conocimiento no solo está personificada en el o la investigadora y la academia, sino en los actores con quienes se trabaja, portadores de saberes, representaciones y soluciones a los problemas que les refieren. De alli que no considerábamos que nuestro ingreso a la escuela secundaria se realizaría mediante la figura clásica de clase o conferencia, ya que un referente comunitario de una organización del barrio nos habia advertido: "la gente no quiere que vengan a darles talleres, quieren ser escuchadas y que se queden". Desde este momento, teníamos claro que el formato a construir surgiría en vínculo con las adolescentes y sus ideas para que llegara a ser ameno e interesante, ya que su asistencia a dicho encuentro sería voluntaria. No podia tratarse de la clase magistral tradicional.

En este orden de ideas describiremos tres momentos importantes en este proceso que hicieron posible la creación de un espacio propicio para hablar de las preguntas y las problemáticas que se les presentan a las adolescentes estudiantes de la escuela secundaria en mención. En primer lugar, abordaremos la importancia de la IAP y el legado de Orlando Fals Borda para el trabajo a realizar con las adolescentes; en segundo lugar, narraremos las negociaciones que fueron necesarias para participar en un espacio como el de una escuela con una fuerte impronta de educación popular y en un tercer momento, reflexionaremos acerca de los retos del feminismo ante los estereotipos que se presentan sobre las adolescentes y los que ellas mismas reproducen.

\section{EL COMPROMISO EN ORLANDO FALS BORDA}

Orlando Fals Borda se convierte en un referente de la IAP a raíz de su vocación por ampliar los ámbitos de investigación de la sociología de su tiempo, acercándose a aquellas dinámicas populares que estuvieron por fuera de los intereses académicos de la Colombia de aquel entonces. Desde su mirada, la historia que se habia escrito en Colombia era la historia sobre las elites, que dejaba de lado la de los pueblos marginados por desinterés de parte de la academia o porque estos se habrian negado a darles información a raíz de la desconfianza hacia el ámbito académico. Las luchas campesinas, indígenas y sindicales fueron observadas con interés por Fals Borda y su equipo académico, quienes se 
De Prácticas y discursos

Universidad Nacional del Nordeste

Centro de Estudios Sociales

Año 8, Número 12, 2019, Octubre

ISSN 2250-6942
LOS DERECHOS SEXUALES EN LA ESCUELA: ESCUCHA, DINAMMICA Y ENCUENTRO UNA EXPERIENCIA DE INVESTIGACIÓN ACCIÓN PARTICIPATIVA

SEXUAL RIGHTS AT SCHOOL: LISTENING, DYNAMICS AND ENCOUNTER A PARTICIPATORY ACTION RESEARCH EXPERIENCE

acercaron a las regiones periféricas del país con el fin de visibilizar procesos históricos no narrados que lograsen poner en jaque los poderes hegemónicos:

El propósito de casi todos mis trabajos ha sido claramente político en el buen sentido del concepto: quería informar y enseñar sobre las realidades encontradas a través de investigaciones interdisciplinarias en el terreno, con el fin de llevar a los lectores, a las masas y a sus dirigentes a actitudes y actividades capaces de cambiar la injusta estructura social existente, especialmente en los campos. (Fals Borda, 2013: 19)

Su definición de la ciencia como un conocimiento que responde a la resolución de necesidades concretas y que se divide en un conocimiento práctico, táctico y estratégico, nos permite comprender que más allá de la necesidad que el autor veía en dar a conocer los relatos y las vivencias de los pueblos con los cuales intercambió, existía en él un interés profundo por la transformación social en el que la noción de compromiso dotaba de sentido a la labor del investigador. Fals Borda entendía el compromiso como una acción o actitud del intelectual a partir de su pertenencia a la sociedad, caracterizada por la renuncia a una posición de simple espectador, poniendo su conocimiento a disposición de los sectores populares. Para ello se inspiró en el aporte de Jean Paul Sartre y su reflexión sobre la noción de compromiso:

La idea sartriana de engagement, como se sabe, es la que más se acerca al concepto de "compromiso" que queremos definir para la sociología de la crisis: es la acción o la actitud del intelectual que, al tomar conciencia de su pertenencia a la sociedad y al mundo de su tiempo, renuncia a una posición de simple espectador y coloca su pensamiento o su arte al servicio de una causa. (Fals Borda, 2013: 188).

Este compromiso no forma parte de las obligaciones del investigador si no que es su decisión inscribirse en un paradigma que contemple al otro $u$ otra desde una relación equivalente en donde cada uno de los actores dispone de un saber valioso para circular y compartir. Nuestra perspectiva de la extensión consideró como primordial el intercambio de saberes rechazando la postura que defiende una transmisión unidimensional por parte de la universidad hacia los territorios con los que trabaja. De alli nuestra elección metodológica (IAP), la cual permite vincular escenarios aca- 
De Prácticas y discursos

Universidad Nacional del Nordeste

Centro de Estudios Sociales

Año 8, Número 12, 2019, Octubre

ISSN 2250-6942

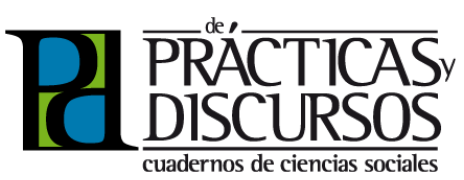

démicos y territoriales rescatando los conocimientos previos de lo situado al rechazar la idea de la superioridad académica según la cual la universidad es la fuente principal de conocimiento.

Hablar en su propio lenguaje y con sus narraciones facilitaría el proceso comunicativo eliminando el "tono" de superioridad de ciertos modos académicos. Así "sobreponer a ellos otra forma de pensar, que implica nuestro lenguaje, nuestra estructura y nuestra manera de actuar, despierta una reacción natural. Una reacción de defensa ante el "invasor" que amenaza romper su equilibrio interno" (Freire, 1973: 32).

Orlando Fals Borda nos invita a repensar el rol de la extensión y a cuestionar su propia categoría. La crítica a la posición de la universidad como ámbito por excelencia, productor de conocimiento, nos lleva a ser partícipes de experiencias sociales de las que emanan saberes transformadores que merecen ser expresados, compartidos y narrados, no solo desde la lógica del investigador que va al terreno para apropiarse del conocimiento, sino en un proceso de ida y vuelta. La idea de "Universidad Participante" de Fals Borda resalta que "la esencia de la investigación participativa no radica necesariamente en la acción, como lo enfatizan las definiciones, sino en la naturaleza y el contenido del lenguaje empleado en la vivencia realizada, esto es, en la información, el diálogo y las modalidades que toma el contacto intersubjetivo del proceso creador" (Fals Borda, 2009: 316). Es este un proceso inacabado, en construcción, que deberá instalarse dentro de las lógicas universitarias y que sin dudas puede ser fundamental para nutrir los espacios de debate en los que se conciban propuestas cercanas a la IAP como metodología válida para la investigación.

Recordar la fuerza social y académica que despertó esta perspectiva de trabajo a partir de la década de los 60 es importante ya que muchos intelectuales se sintieron convocados por esta premisa al sentir que tenían una función activa en la sociedad. Esta forma de entender la ciencia contrasta con muchas de las existentes en la actualidad, en las que puede parecer peligroso para la objetividad en la investigación el hecho de vincularse políticamente. Sobre esto, Pierre Bourdieu nos dice:

Y todo esto ocurre como si las censuras cada vez más apremiantes de un mundo científico cada vez más preocupado de su autonomía (real o aparente) se impusieran cada vez más rigurosamente a los investigadores que, para merecer el título 
De Prácticas y discursos

Universidad Nacional del Nordeste

Centro de Estudios Sociales

Año 8, Número 12, 2019, 0ctubre

ISSN 2250-6942
LOS DERECHOS SEXUALES EN LA ESCUELA: ESCUCHA, DINÁMICA Y ENCUENTRO

UNA EXPERIENCIA DE INVESTIGACIÓN ACCIÓN PARTICIPATIVA

SEXUAL RIGHTS AT SCHOOL: LISTENING, DYNAMICS AND ENCOUNTER

A PARTICIPATORY ACTION RESEARCH EXPERIENCE

de sabios, deben sacrificar en sí mismos la política, y abandonar al mismo tiempo la función utópica a los menos escrupulosos y a los menos competentes de entre ellos a los hombres políticos y a los periodistas. Yo creo que nada justifica esta abdicación cientista, que arruina la convicción política, y que el momento ha llegado en que los sabios deben intervenir en la politica, con toda su competencia, para imponer utopías fundadas en verdad y en razón. (Fals Borda, 2013: 34)

En la obra de Fals Borda queda claro el interés por rescatar los relatos de los conocimientos ancestrales en expresiones como el folclor, la historia, la narración oral, los mitos y la música. A su vez, reconocía la importancia de la creatividad del investigador o investigadora puesta en escena a la hora de realizar la devolución a las comunidades a través de diferentes métodos como los cómics diseñados por campesinos para aquellos que no sabían leer. Se escribieron textos como Historia doble de la Costa (1979) en los cuales quedó plasmada la investigación científica sociológica en una parte del texto y en la otra, los materiales gráficos para las personas que no hacian parte de la academia y no sabían leer. En su parte más descriptiva y literaria, los textos estaban compuestos por poemas, fotografias, mapas, dibujos, pinturas y canciones populares, y del otro lado había una mayor complejidad teórica. Se trató de una apuesta por tender un puente entre la investigación y la sociedad.

El trabajo popular se realizó mediante audiovisuales, filminas, transparencias, grabaciones, conjuntos musicales y dramáticos, y a la par la transmisión del conocimiento científico se generó con la formación de cuadros políticos partiendo de aquellos que tenían más experiencia y habilidades para este tipo de trabajo, para que a su vez se encargaran de terminar el proceso de formación en el resto de la población.

Para Fals Borda, el quehacer científico y cultural debía contener animación, creación e innovación para alcanzar una conexión con el lenguaje popular, de alli que su propuesta de compromiso haya servido de inspiración teórica para el trabajo con adolescentes de una escuela secundaria. Ante la pregunta que alguna vez le hicieran sobre el mayor hallazgo en su propuesta investigativa, Fals Borda respondió:

La insistencia en que teoría y práctica debían ser juntas, no separadas como etapas o dos momentos separados, distintos, sino que se hicieran un ritmo interpretativo, pero de 
De Prácticas y discursos

Universidad Nacional del Nordeste

Centro de Estudios Sociales

Año 8, Número 12, 2019, Octubre

ISSN 2250-6942

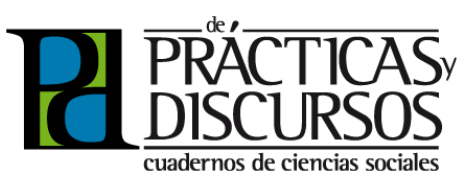

un proceso común, un proceso único. Que ese ritmo fue lo que llamamos ritmo reflexión y acción. Fue como un semillero que después se desarrolló en la práctica y en los efectos concretos, en la aplicación del conocimiento. Fue la diferencia radical con la academia. Porque la pregunta básica era: ¿Para qué el conocimiento y para quiénes va el conocimiento? Esas preguntas no se las hacía la academia. (Fals Borda, 2013: 38)

En el proceso con la escuela técnica fue fundamental partir de una posición ética en la que, más allá de la información encontrada en el espacio con las estudiantes susceptible de ser usada para fines investigativos, lo más importante era brindarles herramientas a las estudiantes para actuar ante situaciones de violencia, discriminación y desigualdad de género. Además de los objetivos investigativos que rigen los intereses de la universidad, se hace fundamental ubicarse en un contexto y reconocer cuáles son aquellas problemáticas más relevantes y persistentes, y asumir una posición de escucha y acciones encaminadas a revertir aquellas problemáticas. Esto solo es posible si se trabaja en conjunto con las estudiantes reconociendo sus preocupaciones e intereses y sumando al espacio aquellos problemas ante los que ha venido luchando el movimiento de mujeres. En su investigación sobre educación sexual integral en las escuelas, Graciela Morgade (2016: 90) nos dice: "la autoridad o más bien la autorización, surge de una compleja interrelación de sujetos, prácticas, métodos y técnicas que se triangulan [...] teniendo en cuenta que "todxs saben", "todxs actúan" y "todxs resignifican" las políticas de educación sexual".

Una de las propuestas de Fals Borda era que las universidades no se agruparan más por facultades, sino por problemas sociales para que el conocimiento tuviera un fin creativo y resolutivo, y no estuviera enquistado sobre sí mismo. Más que buscar la concreción de esta propuesta, sí estuvo claro desde el equipo de extensión que la investigación y la acción debian ir de la mano renunciando a fines y métodos extractivistas en los cuales la sociedad se siente usada y abandonada cuando el investigador consigue sus objetivos, como nos lo advirtieron desde algunas organizaciones territoriales. La noción de compromiso y de escucha debe primar y orientar el trabajo con comunidades sin ser vista como filantropía o solidaridad, ya que se trata de una relación de reciprocidad en que la academia renuncia a su cómoda representación de lugar autorizado de saber por sobre los demás y encuentra 
De Prácticas y discursos

Universidad Nacional del Nordeste

Centro de Estudios Sociales

Año 8, Número 12, 2019, Octubre

ISSN 2250-6942
LOS DERECHOS SEXUALES EN LA ESCUELA: ESCUCHA, DINÁMICA Y ENCUENTRO UNA EXPERIENCIA DE INVESTIGACIÓN ACCIÓN PARTICIPATIVA

SEXUAL RIGHTS AT SCHOOL: LISTENING, DYNAMICS AND ENCOUNTER A PARTICIPATORY ACTION RESEARCH EXPERIENCE

una actitud de respeto en esa relación. Fals Borda nos invita a abordar estos terrenos, a realizar un ejercicio académico político desde alli y a renunciar al cómodo lugar de espectador:

Por eso yo he insistido en que la IAP no se debe enseñar en una clase, sino salir al terreno y dándole continuidad en el tiempo, no estar sujeta a las reglas formales de la academia porque eso contradice toda su filosofía. Si la universidad se compromete con los profesores a mantener la continuidad del trabajo en terreno, está bien; y esa es la primera regla que yo he puesto para lo que ahora se llama universidad participante, que es distinto a extensión universitaria. (Fals Borda, 2013: 41)

\section{LOS VINNCULOS ENTRE LA UNIVERSIDAD Y LA ESCUELA: UNA RELACIÓN A CONSTRUIR}

A pesar de que se trata de dos espacios que parecieran uno, ya que se supone que este último hace parte del primero, ambos lugares tienen sus propias lógicas y dinámicas que los vinculan y los diferencian. La escuela técnica se creó con el propósito de abrir las puertas a la población que habita en el territorio en el que se encuentra la universidad, principalmente José León Suárez, barrio al que pertenecen las estudiantes de la escuela técnica. Defender este tipo de proyecto no es un hecho uniformemente aceptado, ya que en el medio existen representaciones sociales que van desde pensar que los estudiantes de una escuela universitaria deben ser de élite, tener los mejores promedios y trayectorias (como lo expresó una docente de la Facultad) o apostar por una vinculación territorial en donde la trayectoria de los estudiantes no sea lo fundamental, sino la integración social que resarce derechos y permite una relación capaz de crear conocimiento. Este proyecto arraiga una impronta popular en la medida en que la universidad no determina el modo de construir la escuela, entendiendo que su conocimiento no es el único y de allí la relación que se creó con las organizaciones sociales para que la iniciativa no diera respuesta solamente a la función educativa, sino que respondiera a las problemáticas propias de un contexto barrial adverso. Esta iniciativa surge a través de la sumatoria de diferentes actores, lo que le da al proyecto de escuela un matiz más colectivo y popular.

Cuando desde el programa de extensión del Idaes buscamos es- 
De Prácticas y discursos

Universidad Nacional del Nordeste

Centro de Estudios Sociales

Año 8, Número 12, 2019, Octubre

ISSN 2250-6942

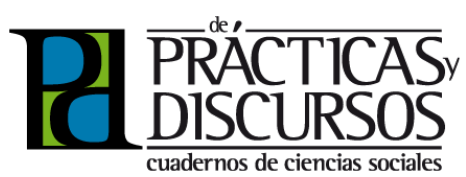

tablecer un vínculo con la escuela, fue necesario romper con una serie de prevenciones disciplinares. A raíz de las conversaciones con referentes de organizaciones sociales o con directores de la escuela o docentes, comprendimos que se referían a nosotros con un tono desconfiado que evocaba un imaginario del investigador interpretado tan solo como un "académico" ajeno a la realidad social, apolítico y sin compromiso. Para romper con este esquema, nuestro argumento en las reuniones de acercamiento se dirigió a comunicar que teníamos experiencia en trabajo con organizaciones sociales a lo que en una ocasión uno de los coordinadores nos respondió: "Es muy diferente trabajar con una organización a trabajar en una escuela". Desde el inicio se hizo evidente un prejuicio en el que los de afuera no podríamos comprender ese lenguaje propio de los sectores populares, de la adolescencia, sus problemas y sus deseos. De este modo asumimos una actitud de escucha y de recepción por sus propuestas como puerta de entrada para iniciar un trabajo con las adolescentes. Se trataba de un desafío interesante porque teníamos claro cuál debería ser nuestro aporte al espacio como investigadoras y feministas, pero no contábamos con formación pedagógica ni lúdica para trabajar con adolescentes. Veníamos de una formación académica que no prioriza la pedagogía ni la lúdica para trabajar en espacios colectivos, de manera que quedaba claro que esta intervención debía realizarse en compañía de docentes y directivos. Nos identificamos con la postura académica política de Graciela Morgade (2016: 84) cuando se definen como:

investigadoras universitarias que rechazan la definición de "autonomía" de la ciencia como el rechazo a la influencia de los movimientos políticos, con militancia en la temática $y$ reconocimiento de los valores que encarnan que, más que sostener una vigilancia epistemológica centrada en silenciarlos o controlarlos, llevan una vigilancia tendiente a hacerlos explícitos para construir los modos metodológicos y técnicos de construcción del "dato" y su interpretación; profesorasinvestigadoras con una importante apropiación de su propia condición de género, empatía con lxs jóvenes, incomodidad con el sistema, interés por profundizar su propio conocimiento a partir de los aportes del equipo de la universidad; profesoras-investigadoras e investigadoras universitarias militantes en diferentes grados que nos "encontramos" en una tarea común, es decir, formación y convicciones previas. 
De Prácticas y discursos

Universidad Nacional del Nordeste

Centro de Estudios Sociales

Año 8, Número 12, 2019, Octubre

ISSN 2250-6942
LOS DERECHOS SEXUALES EN LA ESCUELL: ESCUCHA, DINÁMICA Y ENCUENTRO

UNA EXPERIENCIA DE INVESTIGACIÓN ACCIÓN PARTICIPATIVA

SEXUAL RIGHTS AT SCHOOL: LISTENING, DYNAMICS AND ENCOUNTER A PARIICIPATORY ACTION RESEARCH EXPERIENCE

Habiendo sobrepasado el obstáculo inicial que se produce cada vez que se teje una relación, se inició un conocimiento con los y las diferentes docentes que tenían algún interés en el tema. Algunas de ellas tenian trayectorias de militancia feminista y habian realizado una encuesta sobre las dudas de los y las estudiantes en torno a su sexualidad. Un ejemplo de ello fue la docente de Biología, quien había sido educadora de la Ley de Educación Sexual Integral (ESI), junto con otras docentes que habian detectado relaciones de desigualdad entre hombres y mujeres en la escuela, en las cuales las adolescentes eran insultadas y rebajadas frecuentemente por sus compañeros por su condición de género. El diálogo con cada una de ellas aportó a la construcción del espacio ya que con sus percepciones sobre los problemas y necesidades de las estudiantes fuimos vislumbrando cómo podría constituirse.

Las docentes y estudiantes expresaron su interés en que nuestro acompañamiento sobrepasara el espacio del taller pasando más tiempo con las estudiantes en espacios como los del almuerzo y en otras actividades extracurriculares. Para las docentes, es fundamental construir un vínculo fuerte con las estudiantes que permita la creación de una relación de confianza y que posibilite que las adolescentes tengan otras referentes diferentes a las familiares y las docentes para hablar de sus problemáticas. A su vez, esa contención permite que la adolescente se sienta acompañada por la institución y que en ella haya un lugar en el que no solo se estudia, dado que su contenido político busca la contención integral de la adolescente. Estos lazos no se tejen solamente con las jóvenes, se pretende que estos vínculos se extiendan a las familias y al barrio, que la escuela logre ser un referente ante las problemáticas de marginalidad social y se constituya en una alternativa de transformación. Una propuesta similar nos hace Claudia Korol (2007: 20):

La pedagogía planteada en nuestras búsquedas reúne en su metodología el diálogo, el estudio, las prácticas, la reflexión sobre las mismas, el juego, el deseo, el encuentro, el abrazo, la caricia. El análisis particular y el universal pueden encontrarse en un mismo proceso con la exploración de los sentidos y de los sentires. El saber académico dialoga con el saber popular. El intelectual del movimiento popular interactúa con el intelectual de la Academia. 
De Prácticas y discursos

Universidad Nacional del Nordeste

Centro de Estudios Sociales

Año 8, Número 12, 2019, Octubre

ISSN 2250-6942

\footnotetext{
${ }^{5}$ Decimos aproximadamente ya que su participación no siempre fue constante.
}

${ }^{6}$ Anteriormente habíamos trabajado con un grupo de mujeres de una planta de reciclado desde el equipo de extensión del ldaes y esta experiencia nos mostró la comodidad y confianza con que las mujeres pudieron expresarse acerca de situaciones de violencia o vulnerabilidad. Pasado un tiempo se sumaron algunos hombres a los talleres de la escuela.

${ }^{7}$ En 2015 ya se habían realizado actividades puntuales de las que tuve la oportunidad de participar para trabajar el tema a través de técnicas como las de teatro del oprimido, impulsadas por organizaciones barriales, con el fin de incidir activamente en la escuela.
Una vez establecido el vínculo a partir del interés del proyecto de extensión y de la necesidad que algunos de los actores de la escuela reconocieron en profundizar en el tema, pudimos comenzar a trabajar a través de reuniones semanales de aproximadamente dos horas con un grupo de tercer y cuarto año de secundaria, conformado por aproximadamente ${ }^{5}$ quince adolescentes en edades que oscilaron entre los 14 y los 16 años.

Decidimos que el grupo estaría conformado por mujeres considerando que esto posibilitaría la creación de un espacio de confianza en que pudieran expresarse con mayor libertad y sin vergüenza al hablar de temas como la sexualidad que para la sociedad han sido vistos tradicionalmente como tabú ${ }^{\text {. }}$

\section{LOS ENCUENTROS CON LAS ADOLESCENTES}

El primer día que visitamos la escuela, con el objetivo de contarles a las adolescentes nuestro propósito de trabajar la temática de género, se caracterizó por el silencio y la timidez a diferencia de encuentros anteriores en actividades puntuales de sensibilización sobre la problemática de género y la promoción de derechos $^{7}$. Iniciamos contándoles sobre la existencia de una consejería universitaria en salud sexual y reproductiva, donde podian expresar sus dudas más personales en torno al tema si tenían vergüenza de hablar con sus familias o compañeras. Enfatizamos en la importancia de la identificación de la violencia de género y las luchas por erradicarla, a lo que una de las estudiantes con mayor liderazgo respondió que no se encontraba interesada en asistir porque en la actualidad no pretendía tener sexo y porque cuando lo decidiera, tendría a quién preguntárselo.

Ella argumentaba que habia chicas "buenas" y chicas "malas" según los hombres -es decir, sus amigos- aclaró, y que a ella le interesaba escucharlos para orientarse, para saber cómo comportarse y no parecer una "zorra". Fue la única que habló, las demás se quedaron silenciosas y un poco avergonzadas por abordar la temática. En el segundo encuentro hubo una mayor distención y participación, y a pesar de que el tema inicial no tenía nada que ver con la diversidad sexual, en algún momento empezaron alusiones hacia las "torti" como se suele nombrar a las lesbianas. Se escucharon apreciaciones como: "iQué asco las torti!" y mencionaban que conocian a algunas de su barrio que jugaban a la pelota. Una de las adolescentes contó que una de sus compañeras de fútbol 
De Prácticas y discursos

Universidad Nacional del Nordeste

Centro de Estudios Sociales

Año 8, Número 12, 2019, 0ctubre

ISSN 2250-6942
LOS DERECHOS SEXUALES EN LA ESCUELA: ESCUCHA, DINÁMICA Y ENCUENTRO

UNA EXPERIENCIA DE INVESTIGACIÓN ACCIÓN PARTICIPAIIVA

SEXUAL RIGHTS AT SCHOOL: LISTENING, DYNAMICS AND ENCOUNTER A PARIICIPATORY ACTION RESEARCH EXPERIENCE

era "torti" y que ella la aconsejaba -desde sus representaciones de lo que debía ser una mujer- ya que su amiga contaba con el apoyo de su madre mientras su papá la rechazaba. Para la estudiante, el ámbito del fútbol femenino del que ella participaba era un espacio común para las "tortis", en el que un ambiente deportivo estaba estrictamente relacionado con la masculinidad, y de alli se seguia que las mujeres que participaban de él tenían alguna preferencia por dicha identidad sexual. Fue muy interesante que la docente de Física las interpelara constantemente diciéndoles que ella era una "torti" y que tenía un novio, esto con el fin de perturbarlas y de darle un poco de complejidad a un fenómeno social que ha sido tradicionalmente entendido desde reglas heteronormativas rígidas. Las estudiantes, en general, manifestaron repulsión hacia el lesbianismo, la mayoria de ellas declaró fastidio diciendo que no querian tener amigas "torti" y me interpelaban con sorpresa cuando les hablé con naturalidad sobre mis amigas lesbianas. Preguntaron qué pensaba de ellas, a lo que les dije que tenía muchas y que las quería. Su reacción fue de sorpresa. Fue interesante que al momento en que la docente declara su lesbianismo las estudiantes no le prestaron mucha atención porque ya habian escuchado su postura y la reconocian como un personaje cercano. Alguna dijo: "porque esta es una loquita", pero no se le prestó demasiada atención ni se escandalizaron. Se manifestó un repudio hacia lo desconocido, hacia la lesbiana desconocida, pero cuando se trata de personas cercanas que hacen parte de su cotidianidad, las adolescentes desplazan sus prejuicios y lo asumen como algo más natural.

A su vez, indagamos en que muchas veces las personas heterosexuales pensaban que compartir con una lesbiana era peligroso porque sentian que iban a ser objeto de su deseo, lo que evidentemente no era así porque las lesbianas tenían gustos diversos y no necesariamente tenian que gustarles todas las mujeres. Esta situación provocó que la docente que se declaraba lesbiana le dijera a una de ellas: "Sí, me gustan las mujeres, pero vos no me gustás", hecho que interpeló directamente a las chicas acerca de una visión peligrosa y perversa de la diversidad sexual. Existe un temor, un tabú, una negación, pero al parecer se trata de un hecho ominoso. Un par de años atrás, habiamos trabajado en un espacio más puntual y las estudiantes nombraban como "torti" a una de sus compañeras que se caracterizaba por su timidez y por tener el cabello muy corto. Se extendía un estereotipo y un señalamiento 
De Prácticas y discursos

Universidad Nacional del Nordeste

Centro de Estudios Sociales

Año 8, Número 12, 2019, Octubre

ISSN 2250-6942
8 Ver: http:/ /www.t elam. com.ar/notas/201606/ 151051- tiroteo- muertos- club-gay-orlando.html sobre una adolescente solo por su estética, ya que nunca había hablado de sus preferencias sexuales. Se dejaba ver un prejuicio, un señalamiento y una burla a posibles identidades sexuales diferentes. Sin embargo, resulta de vital importancia el cuestionamiento permanente que las y los docentes realizan a los estereotipos de género en tanto estos son desalojados de su lugar de verdad y sufren interpelaciones que le dan a la adolescente un mayor ámbito de posibilidades para interpretar como lo dirá Paula Fainsod (2007):

Un aspecto altamente significativo para pensar el lugar de las escuelas en la configuración de subjetividades femeninas y masculinas es aquel que refiere a los procesos de clasificación y nombramiento que en ellas tienen lugar. Las expectativas que ponen en juego los y las docentes se transforman, para los propios alumnos y alumnas, en actos de nombramiento que los atraviesan en la construcción de su auto-estima e identidad impactando en su trayectoria escolar y social.

De manera que estos juegos en la identidad producen una pluralidad de sentidos en el espacio áulico que contrasta las dinámicas barriales, familiares y sociales con las que los y las adolescentes se han venido identificando. Esta producción de sentido también se relaciona con una apertura de derechos a ser ejercidos, reconocidos e incorporados.

En el aula, el trato se caracterizó por tener un tono fuerte, en el que las experiencias sexuales de algunas de sus compañeras con los chicos eran divulgadas constantemente en presencia de las demás y frases como "zorra de mierda" y "negra de mierda" fueron frecuentes en sus discusiones. Sin embargo, estas frases no pasaron desapercibidas por las docentes. Ellas se encargaron de problematizar estos imaginarios cuestionándoles por su propia clase social y raza para desarmar la insensatez del comentario. Se trataba de rebajarse y dejar a la otra sin argumentos, pero en un tono de burla que no fuera contradictorio con la amistad. Esta sesión tomó un giro muy interesante ya que coincidió con el asesinato masivo en EEUU en un bar gay8. La temática nos permitió reflexionar sobre la peligrosidad de sostener prejuicios homoodiantes que pueden incluso motivar la eliminación de la diferencia. Cuando interpelamos a las adolescentes sobre la similitud de sus 
De Prácticas y discursos

Universidad Nacional del Nordeste

Centro de Estudios Sociales

Año 8, Número 12, 2019, Octubre

ISSN 2250-6942
LOS DERECHOS SEXUALES EN LA ESCUELA: ESCUCHA, DINAMMICA Y ENCUENTRO UNA EXPERIENCIA DE INVESTIGACIÓN ACCIÓN PARTICIPATIVA

SEXUAL RIGHTS AT SCHOOL: LISTENING, DYNAMICS AND ENCOUNTER A PARTICIPATORY ACTION RESEARCH EXPERIENCE

prejuicios con los de los argumentos de alguien que asesinó por intolerancia homosexual, manifestaron que ellas nunca harían algo así, es decir, que sus juicios nunca llegarían a acciones de violencia. Fue un escenario que permitió aterrizar la situación y reflexionar sobre las consecuencias y el peligro de las ideologias dogmáticas.

Otras adolescentes más silenciosas no le dieron la misma importancia al lesbianismo, esbozaron argumentos de libertad y autonomía en la elección de la identidad sexual. Una de las estudiantes habia tenido una experiencia de asistir a una iglesia en la que no se juzgaba la diversidad sexual y se alentaba a respetarla, entonces tenía una mayor apertura ante el tema.

En esta oportunidad, dos chicos irrumpieron en el aula y preguntaron sobre qué estábamos hablando, a lo que les contamos lo referido a la masacre de EEUU, mientras una de ellas me retó diciéndome que nos habiamos comprometido a no contar las intimidades de las demás compañeras, lo que me sirvió para aclararles el sentido de este pacto. Una de ellas lo interpeló preguntándole qué pensaba de las "torti", a lo que en un primer momento hizo un gesto de que no le importaba y en un segundo momento le achacó a quien más aversión había declarado sobre las lesbianas: "si vos sos torti". Lo que estaba en juego era producir una identidad respetable para el resto de sus compañeras, alcanzar un espacio de reconocimiento y no salirse de los parámetros que construyen al sujeto "normal". Esta necesidad de aceptación tan fuerte en los colectivos es uno de los rasgos que permite construir identidad y a los que las adolescentes se aferran. En este sentido, Honneth (1992: 188-189) dirá:

debemos nuestra identidad... a la aprobación y el reconocimiento que recibimos de otras personas. (Conceptos negativos tales como insulto o degradación) están relacionados con expresiones de falta de respeto, con la denegación de reconocimiento. (Estos conceptos) se emplean para caracterizar una forma de comportamiento que no representa una injusticia solamente porque constriña la libertad de acción de los sujetos o les inflija un daño. Por el contrario, dicho comportamiento resulta dañino debido a que perjudica a estas personas en su comprensión adecuada de sí mismas, una comprensión adquirida por medios intersubjetivos. 
De Prácticas y discursos

Universidad Nacional del Nordeste

Centro de Estudios Sociales

Año 8, Número 12, 2019, Octubre

ISSN 2250-6942

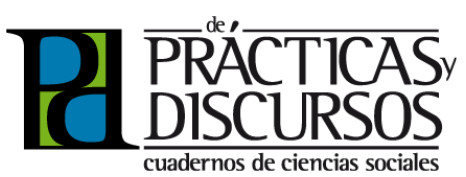

A su vez, las estudiantes cuestionaban permanentemente su feminidad en tanto se alejaban del estereotipo de decencia y fidelidad o cuando parecian más cercanas de los estereotipos masculinos. Esto se expresa en dos de los insultos más comunes escuchados en la escuela: "puta" o "marimacha".

En este tipo de espacios, que no dejan de ser espacios de poder, no solo se juegan las identidades, también se establece el respeto que se puede llegar a obtener. Esto nos permite pensar que detrás de todo el ahínco con el que se juzga el lesbianismo hay un temor, un miedo al rechazo, a no ser parte del orden y a ser señalada como diferente y moralmente enjuiciable, más aún cuando los demás emiten juicios que cuestionan la identidad. En este sentido, tal como dice Morgade (2016: 25):

las escuelas no solo no son neutrales desde la perspectiva de género sino que tienden, en tanto aparatos ideológicos, a sostener y reproducir los sentidos predominantes en los sectores hegemónicos: tiende a permanecer una división en los lugares atribuidos sexogenéricamente en la reproducción y en la participación económica y política (manteniéndose de este modo una estructuración patriarcal de la sociedad), y tienden a ser invisibilizadas todas las formas de experiencia humana que no se expresan a través de los modos heterosexuales de vivir el cuerpo.

\section{CREATIVIDAD Y JUEGO}

La presencia de las docentes o coordinadoras que nos acompañaron fue fundamental porque ellas participaron y opinaron sobre los contenidos, sobre los futuros encuentros y sobre todo de la dinámica, que es el mayor de los retos: que las adolescentes encuentren interés en el espacio, que no sientan que asistir es una obligación y que consideren que en el taller se pueden construir proyectos que las motiven. El desafio del espacio sería construir herramientas que las doten de fuerza para responder ante situaciones dificiles.

De esta manera, continuamos trabajando con el grupo temas alusivos a la salud y los derechos sexuales, la anticoncepción y la violencia de género. Lejos de tratarse de una clase, fueron charlas más personales, distendidas, en las que las adolescentes rieron y buscaron que fuese un espacio divertido. Entonces fue apropiado utilizar un texto, reflexionar y luego pasar a charlas más 
De Prácticas y discursos

Universidad Nacional del Nordeste

Centro de Estudios Sociales

Año 8, Número 12, 2019, 0ctubre

ISSN 2250-6942
LOS DERECHOS SEXUALES EN LA ESCUELA: ESCUCHA, DINÁMICA Y ENCUENTRO

UNA EXPERIENCIA DE INVESTIGACIÓN ACCIÓN PARIICIPATIVA

SEXUAL RIGHTS AT SCHOOL: IISTENING, DYNAMICS AND ENCOUNTER A PARIICIPATORY ACTION RESEARCH EXPERIENCE

relajantes y hablar de sus preferencias televisivas, su interés por las películas de terror, la macumba y el baile. Se trató de ingresar a su mundo e intereses para poder establecer una relación. Las adolescentes siempre demandaron actividades distendidas, de forma que si leíamos un texto acerca de la violencia de género reflexionábamos un rato sobre él, pero sabiamos que debiamos pasar prontamente a otra actividad. Por ello, no siempre fueron suficientes las cartillas de ESI del ministerio y debimos pensar juegos y jugar a los que ellas proponían con el fin de afianzar el vínculo. En una oportunidad, las coordinadoras nos invitaron a asistir al baby shower de una de las integrantes del grupo. La escuela en su conjunto creó una actividad para acompañar el embarazo de la estudiante, dándole regalos y compartiendo a través de una fiesta. Para los coordinadores, era fundamental que además de compartir en el espacio del taller, fuéramos creando un vínculo con las adolescentes por fuera de dicho espacio para incentivar la confianza y afianzar el vínculo. Este hecho abre un gran interrogante a la investigación, nos lleva a pensar hasta dónde debemos participar, cómo se operacionaliza el compromiso y cómo se construyen las relaciones. La educación popular ha optado por crear un vínculo más humano y constante que sobrepase la rigidez de los espacios académicos.

Recurrimos a una serie de materiales tales como películas, música, creación de historias, actuación y dibujo para crear un ambiente interesante y dinámico para las estudiantes. Conservamos los escritos e imágenes que se produjeron en el taller. Sin embargo, no siempre cumplimos con el objetivo que nos habiamos trazado inicialmente, muchas veces las adolescentes no querian tratar los temas que habiamos propuesto o no deseaban profundizar en la discusión. Lejos de ser una experiencia frustrante, comprendimos que era un paso para afianzar nuestra relación y el conocimiento mutuo. Reconocer sus intereses y experiencias de vida se volvió fundamental para comprender el contexto del que provenian, en el que podian oscilar entre la sobreprotección o indiferencia de sus familiares, su necesidad de afirmarse en el ambiente escolar $y$, casi siempre, una negativa por parte de sus familiares a que ejercieran sus derechos sexuales y reproductivos. Las docentes nos prevenían sobre la vigilancia de sus madres y la prohibición de usar métodos anticonceptivos, en busca de detener el inicio de su sexualidad.

Identificar este contexto resulta fundamental para la interven- 
De Prácticas y discursos

Universidad Nacional del Nordeste

Centro de Estudios Sociales

Año 8, Número 12, 2019, Octubre

ISSN 2250-6942

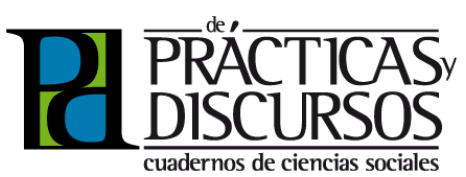

ción. Las dinámicas barriales de las que provienen las adolescentes con quienes trabajamos están caracterizadas por situaciones de escasez económica, patrones familiares no nucleares y barrios carentes de equipamientos. Sin embargo, se cuenta con la fortaleza de habitar un territorio en donde hay una fuerte organización social que erige los intereses del colectivo y en donde la escuela universitaria ocupa un papel fundamental por su compromiso con los sectores populares. Además de ello, la universidad se acerca a este contexto con una pregunta por quién es ese sujeto, pregunta fundamental para el desarrollo de un proyecto educativo, como lo señala Fainsod (2007):

Pensar el tema de las desigualdades sociales y educativas requiere abordar una perspectiva que reconozca las condiciones estructurales que determinan fuertemente a los sujetos que habitan las escuelas o aquellos que están en sus márgenes. Los procesos de fragmentación y exclusión al que las politicas neoliberales subsumieron a vastos sectores sociales, tornan necesaria la pregunta por quiénes son esos sujetos, qué sentidos y expectativas construyen, qué experiencias sociales y escolares construyen en el marco de las transformaciones estructurales de los últimos años. Esta pregunta resulta altamente significativa, además, en un contexto en el cual adquieren legitimidad los discursos que transmutan las desigualdades sociales en naturalezas y/o capacidades de los individuos.

Para responder esta pregunta, fue primordial darle lugar al diálogo incorporando sus propuestas e intereses. En esta búsqueda, nos encontramos con la propuesta de una de las estudiantes de realizar un corto sobre cómo es la vida de una joven de José León Suárez. Para la realización del corto, contamos con la participación de algunas estudiantes de cine y teatro de la universidad que se ofrecieron a poner a disposición sus conocimientos. En este tipo de proyectos resulta fundamental vincular a otros sectores interesados en participar $y$, en este caso, las propuestas artísticas y culturales son necesarias para encontrar otros lenguajes de expresión que puedan convocar a las adolescentes.

\section{CONCLUSIONES}

Es fundamental reflexionar sobre la relación entre conocimiento y práctica política entendiendo que no se trata de términos ex- 
De Prácticas y discursos

Universidad Nacional del Nordeste

Centro de Estudios Sociales

Año 8, Número 12, 2019, Octubre

ISSN 2250-6942
LOS DERECHOS SEXUALES EN LA ESCUELA: ESCUCHA, DINÁMICA Y ENCUENTRO UNA EXPERIENCIA DE INVESTIGACIÓN ACCIÓN PARTICIPATIVA

SEXUAL RIGHTS AT SCHOOL: LISTENING, DYNAMICS AND ENCOUNTER A PARTICIPATORY ACTION RESEARCH EXPERIENCE

cluyentes al remitir a contenidos éticos que pueden servir de guía a la labor de la investigación, dotándola de sentido. La experiencia de Orlando Fals Borda en Colombia nos permite pensar en la pertinencia de tener vigente ideales y principios que remiten a la transformación social sin aislarlos por temor a desertar de las normativas científicas.

La relación entre conocimiento y práctica política también posibilita pensarse como sujetos de transformación capaces de sobrepasar la lectura concienzuda, asumiendo también un rol pedagógico y de escucha ante procesos sociales concretos y en construcción, en donde debe estar presente la reflexión académica y el uso de la creatividad y la imaginación para fortalecer dichos procesos, para llenarlos de contenido y sumar propuestas y actividades concretas. A su vez, estas actividades deben remitir a reflexiones teóricas y políticas capaces de orientar las acciones y de crear ejercicios de ida y vuelta, donde la teoría sea desafiada por la realidad social en la medida en que esta es dinámica, cambiante y exigente de nuevas metodologías para ser atendida.

No basta con recurrir a soluciones anticipadas, como nos sucedió con los cuadernos de ESI del ministerio, ya que a pesar de que son materiales de incalculable valor, sometidos a procesos populares, no siempre cumplen con su función potenciadora; en algunos casos la canción o el cuento propuesto no fue la más conveniente, ya que los jóvenes solicitaron materiales más lúdicos, más llamativos y más cercanos a sus experiencias barriales y de clase social. Este hecho nos remite a pensar en la necesidad de partir de un conocimiento situado, que valore la pluralidad del mundo popular y tenga en cuenta sus diferencias e intereses con otras clases sociales, tal como lo trabajó Fals Borda. Se convirtió, entonces, en un desafío hacer uso de los manuales del ministerio, pero adaptándolos a contenidos más cercanos a sus experiencias.

Este conocimiento situado, interesado en la praxis social, considera permanentemente un desafío poner en funcionamiento la teoría, cómo someterla al calor de la experiencia, cómo adaptarla y readaptarla para trabajar en contextos concretos como el que nos propusimos crear con las adolescentes. Ante estas preguntas, Fals Borda (2013: 213) sugiere:

Cómo combinar precisamente lo vivencial con lo racional en estos procesos de cambio radical, constituye la esencia del problema que tenemos entre manos. Y éste, en el fondo, es 
De Prácticas y discursos

Universidad Nacional del Nordeste

Centro de Estudios Sociales

Año 8, Número 12, 2019, Octubre

ISSN 2250-6942

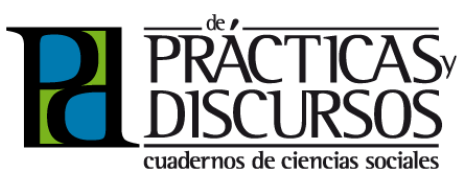

un problema ontológico y de concepciones generales del que no podemos excusarnos. En especial, ¿qué exigencias nos ha hecho y nos hace la realidad del cambio en cuanto a nuestro papel como científicos y en cuanto a nuestra concepción y utilización de la ciencia? Porque, al vivir, no lo hacemos solo como hombres, sino como seres preparados para el estudio y la crítica de la sociedad y el mundo.

Además de la crítica y de la reinvención de la teoría, es justo señalar la necesidad de ejercitar la capacidad inventiva en actividades concretas como las desarrolladas con adolescentes. Venir de formaciones teóricas como la ciencia política y la sociología genera un vacio y un desconcierto a la hora de desarrollar actividades grupales que más que teóricas deben tener un fuerte componente lúdico, pedagógico y creativo, lo que exige una búsqueda de las propuestas desarrolladas por otras disciplinas que se ejercitan más en esta materia con el fin de aprender y adaptar dichas propuestas a nuestras necesidades.

Este interés de trabajo ya refleja en sí mismo un cambio en la mirada de la investigación, un rechazo a los criticados métodos extractivistas denunciados por las comunidades que ya no quieren sentirse herramientas de los científicos sociales. Quizá deseen una mayor participación en los procesos que los convocan, el respeto de sus estrategias y la necesidad de trabajar en conjunto sin sentirse la fuente de conocimiento que es usada y abandonada.

En este sentido, fue fundamental no someter a los adolescentes a una serie de preguntas, a guías de entrevista, ya que nos acercamos a través de talleres, diálogos y charlas en el almuerzo, para conocerlas, exponer nuestros puntos de vista, escuchar los suyos y crear un conocimiento que pueda ser una herramienta para resolver problemas de carácter más cotidiano. Esto quiere decir que, además de reflexionar sobre los derechos de las mujeres, fue fundamental transmitir los derechos que tenian en las salitas a obtener información y métodos anticonceptivos, conocer que la universidad los ofrece, que podemos otorgárselos, acompañarlas en sus decisiones, es decir, efectivizar los derechos.

Alli radica la construcción de confianza, que no significó que nos convirtiéramos en las mejores amigas de las jóvenes, sino en que pudiéramos ser identificadas como personas que pueden resolver una duda, acompañar o indagar más sobre la temática. La sexualidad no es siempre una temática sencilla de abordar. 
De Prácticas y discursos

Universidad Nacional del Nordeste

Centro de Estudios Sociales

Año 8, Número 12, 2019, 0ctubre

ISSN 2250-6942
LOS DERECHOS SEXUALES EN LA ESCUELA: ESCUCHA, DINÁMICA Y ENCUENTRO

UNA EXPERIENCIA DE INVESTIGACIÓN ACCIÓN PARIICIPATIVA

SEXUAL RIGHTS AT SCHOOL: LISTENING, DYNAMICS AND ENCOUNTER

A PARTICIPATORY ACTION RESEARCH EXPERIENCE

¿Cómo hablar de sexualidad cuando algunas no la desean porque quieren postergar su primera experiencia sexual y por lo tanto postergar también la temática? ¿Cómo hablar sobre prevención de embarazo adolescente sin generar incomodidad para quien ya es madre? ¿Cómo hablar sobre el derecho al aborto sin que las adolescentes sientan que queremos pasar por encima de sus convicciones morales o religiosas? Son, sin duda, preguntas que nos formulamos pensando en no pasar por encima de los principios o convicciones de nuestro grupo de trabajo, intentando ser respetuosas de sus deseos y creencias. Estos desafios son interesantes para la investigación ya que no existen respuestas cerradas y precisas, lo que existe es reflexión y adaptación de las propuestas teórico políticas.

A nivel personal, también se presentan desafíos. Cómo actuar en espacios caracterizados por el ruido, la burla y el juego, cómo transmitir un contenido logrando que las adolescentes se interesen, que se trate de un espacio llamativo y lúdico. Dónde encontrar actividades como juegos que sean de su interés. Todas estas preguntas fueron fundamentales para abordar cada sesión del taller, lo que a su vez nos movilizó en la búsqueda de obras de teatro, películas y lecturas que tuvieran que ver con la temática de género y que tuvieran cercanía a su edad y su contexto.

Mi condición de migrante no pasó desapercibida y fue paradójica a la hora de construir una relación con las adolescentes. En un primer momento, había muchos términos y modismos que no podía comprender a raíz de la distancia cultural y, sin embargo, en un segundo momento esta diferencia cultural fue positiva ya que las estudiantes se hallaban interesadas en la tonada y querian imitarla, lo que permitió una relación de interés y de confianza. Hubo momentos en los cuales fue difícil trabajar sobre esta diferencia, ya que funcionó como motivo de burla a través de la imitación y al estereotipar mi nacionalidad. En sintesis, esta condición trajo su provecho y sus dificultades al afianzar la comunicación con las adolescentes y escuchar los estereotipos que se reproducen acerca de la nacionalidad, en mi caso la colombiana.

De otro lado, es fundamental no perder de vista que el trabajo de los docentes de la escuela debe estar motivado por garantizar los derechos de las adolescentes. Informar y publicitar los derechos debe ser una estrategia para combatir la desigualdad que, entre otras, tiene que ver con el acceso a sus derechos sexuales. En algunos casos, en la decisión de los adolescentes prevalecen 
De Prácticas y discursos

Universidad Nacional del Nordeste

Centro de Estudios Sociales

Año 8, Número 12, 2019, Octubre

ISSN 2250-6942

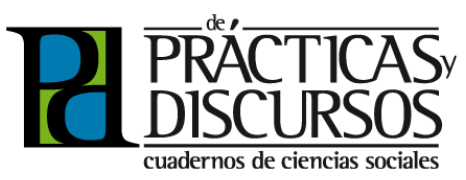

\footnotetext{
${ }^{9}$ Algunas docentes participaban de agrupaciones feministas y ya tenían experiencia en trabajo comunitario abordando la problemática de género. También nos encontramos con una docente que había sido educadora sexual en capacitaciones masivas a docentes del programa nacional Educación Sexual Integral (ESI).
}

las convicciones religiosas o el respeto por la opinión de sus madres ante la decisión de, por ejemplo, un aborto. Ante situaciones como estas, también se encuentran docentes comprometidos que desean acompañar el deseo de las adolescentes y mostrarles un espectro más amplio de posibilidades para que tomen sus decisiones. Es en este sentido que consideramos fundamentales las experiencias de militancia previas de las y los docentes que acompañaron a las estudiantes en sus decisiones relacionadas con la anticoncepción, el aborto o la maternidad?

Comprender que la educación no se termina con el horario de clase y que la contención resulta fundamental para complementar este proceso permitió que las adolescentes se sintieran más acompañadas y que pudieran hablar de temas que en sus casas están vedados, como el de la sexualidad. Por ello, el compromiso entre universidad y escuela resulta fundamental. Reforzar los propósitos de creación de un lenguaje común se torna en un objetivo dificil, pero en el que se debe trabajar para llegar a una retroalimentación que nos lleve a mejores prácticas y a la producción de conocimiento.

A pesar de que la escuela posee una fuerte impronta de educación popular, es necesario que se refuercen las actividades tendientes a sensibilizar y modificar las relaciones de género, ya que para las docentes es visible la desigualdad y violencia hacia las mujeres, y en los encuentros con las estudiantes la naturalización de las relaciones desiguales entre los géneros se hizo evidente. En este sentido, los contenidos de educación popular son insuficientes para crear una escuela transformadora, debe existir el compromiso con la erradicación de todo tipo de desigualdades y las de género no son problemáticas de segundo orden. Con miras a cumplir este objetivo, tanto investigadores, docentes, universidad como la escuela deben comprometerse en aportar a dicha transformación ya que la escuela también está cruzada por historias personales de reproducción del machismo, educación católica y prejuicios que tienden a ver al feminismo como un fantasma más que como una lucha por la igualdad social.

En este mismo sentido, pensar en conexión la educación popular y el género marcó un desafío novedoso en comparación con las problemáticas que se planteaban los investigadores del equipo de Fals Borda de la Colombia del 70. La temática de género no tenía la visibilidad que tiene en la actualidad y la fuerza que tiene el movimiento social en la Argentina. De alguna forma, esto posibilitó que 
De Prácticas y discursos

Universidad Nacional del Nordeste

Centro de Estudios Sociales

Año 8, Número 12, 2019, Octubre

ISSN 2250-6942
LOS DERECHOS SEXUALES EN LA ESCUELL: ESCUCHA, DINÁMICA Y ENCUENTRO UNA EXPERIENCIA DE INVESTIGACIÓN ACCIÓN PARTICIPATIVA

SEXUAL RIGHTS AT SCHOOL: LISTENING, DYNAMICS AND ENCOUNTER A PARTICIPATORY ACTION RESEARCH EXPERIENCE

nuestra propuesta fuera tenida en cuenta en la escuela y que pudiéramos trabajar un problema con amplia visibilidad social, lo que no quiso decir que las adolescentes estuvieran apropiadas del discurso. Consideramos que seguir profundizando en esta conexión es fundamental para que las adolescentes alcancen un mayor conocimiento de sus derechos, para que las escuelas que consideran que la educación popular es un estandarte de la educación no permitan que en sus estructuras el machismo pase desapercibido y se siga trabajando desde ambos frentes para ofrecer a las adolescentes un amplio espectro de posibilidades, pues como lo enuncia Fainsod (2007), la escuela también tiene lugar en la construcción del sujeto: las escuelas no se limitan tan solo a reproducir sus propios mecanismos de distribución, sino que ellas fabrican -o contribuyen a fabricar- actores y sujetos.

Finalmente, debemos destacar nuestra apuesta de trabajo, que utiliza como metodología la IAP, considerando que esta permite construir vínculos más honestos con las comunidades con las que trabajamos, relaciones de ida y vuelta, aprendizajes múltiples, sin que por esto desmerezca en rigurosidad. La ética profesional también se expone en este tipo de vínculos en los que el diálogo de saberes orienta la relación que se produce en la investigación y marca desafíos políticos y de transformación ante desigualdades tan profundas como la que supone el género.

\section{BIBLIOGRAFÍA}

BOURDIEU, P. (2000). Sobre el campo político. Presses universitaires de Lyon. Disponible en : http://200.6.99.248/ bru487cl/ files/BOURDIEU_campo-politico.pdf

FAINSOD, P. (2007). Interrogar las certezas. Embarazos y maternidades adolescentes en la escuela. XXVI Congreso de la Asociación Latinoamericana de Sociología. Guadalajara: Asociación Latinoamericana de Sociología.

FALS BORDA, 0. (1979). Historia doble de la Costa. Bogotá: Carlos Valencia Editores.

(1980). IV. La ciencia y el pueblo: nuevas reflexiones. En La sociología en Colombia: balance y perspectivas. Bogotá: Editora Guadalupe. 
(2009). IV Ciencia y praxis. En Una sociología sentipensante para América Latina. Bogotá: Siglo del hombre editores.

(2013). Ciencia, compromiso y cambio social. Buenos Aires: Colección pensamiento latinoamericano.

Freire, P. (1973) ¿Extensión o comunicación? La concientización en el medio rural. México: Siglo XXI.

HOONETH, A. (1997). "¿De la redistribución al reconocimiento? Dilemas en tomo a la justicia en una época "postsocialista". En Fraser (ed.) lustitia Interrupta: Reflexiones críticas desde la posición "postsocialista". Santa Fe de Bogotá: Siglo de Hombres Editores.

KOROL, C. (2007). Hacia una pedagogía feminista. Géneros y educación popular. La educación como práctica de libertad. Editorial El colectivo, América Libre.

MORGADE, G. (2016). Educación sexual integral. La lupa de la ESI en el aula. Rosario, Argentina: Homo Sapiens ediciones. 